\title{
100. Supplement to S. Tsuboi's Previous Paper on Retardation Determination of a Birefringent Crystal Section
}

\author{
By Seitarô Tsubor, M. J. A. \\ Geological Institute, Faculty of Science, University of Tokyo
}

(Comm. June 12, 1973)

In the paper entitled "A method of determining the retardation of a birefringent crystal section" (S. Tsuboi : Proc. Japan Acad., 40, 1964, 279-284), the present writer described a new device in polarizing microscopy for retardation determination. The procedure there proposed is briefly as follows :

On the stage of a polarizing microscope, put a section S, whose retardation $R_{\mathrm{S}}$ is to be determined, in diagonal orientation; insert between the section and the analyzer a wedge made of a colourless crystal, specially devised for the proposed method, in orthogonal orientation; transmit monochromatic light of wave-length $\lambda_{0}$ from below; observe the row of light and dark bands which is visible in the microscopic field through the analyzer at its appropriate azimuth; locate the central line of symmetry in the brightness distribution there, and bring it to a suitable position (e.g. the center of the microscopic field) by sliding the wedge; rotate the analyzer until the central line becomes completely dark, and read the angle $\theta^{\prime}$ through which the analyzer has been rotated counterclockwise from its initial azimuth, where the vibration direction is $\leftrightarrow$; then, the retardation $R_{\mathrm{S}}$ is obtained by the relation:

$$
R_{\mathrm{S}}=\frac{\theta^{\prime} \lambda_{0}}{\pi}+n \lambda_{0},
$$

where $n=0$, or 1 , or $2, \cdots$.

In the present paper are given some notes supplementary to what were described previously. More particularly it is concerned with a design of construction of the wedge and an improvement in the experimental procedure.

\section{On the Construction of the Wedge}

The important accessory instrument required in the method proposed is a specially devised wedge, by means of which a continuous series of varied retardation can be obtained. No detailed description was given of the construction of the wedge in the previous paper, 
where only the principle of the method was presented. For a practical application of the method it is essential to have a well-designed, efficient wedge. A compound wedge constructed on the following design will be suitable for use in omnifarious cases.

Two plates are cut from a colourless, transparent crystal of quartz,- - a wedge-shaped one M and a plane-parallel one N, as in Fig. 1. In plate $\mathrm{M}$, the crystallographic axis $\mathbf{c}$ of quartz is oriented parallel to the elongation, while in plate $\mathrm{N}$, perpendicular to the elongation. The compound wedge is constructed by superposing plate $\mathrm{M}$ on plate

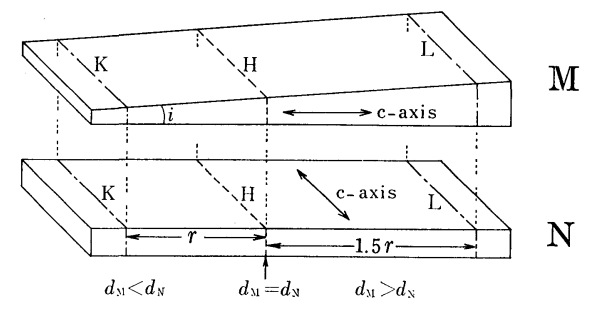

Fig. 1. Wedge-shaped plate and plane-parallel plate of quartz for constructing the compound wedge.

$\mathrm{N}$ and cementing them. The inclination angle of the wedge (the angle between the two planes of the wedge), designated as $i$, is given by the relation:

$$
\tan i=\frac{0.1 \mathrm{~mm}}{2 r},
$$

where $2 r$ is the real length on the wedge along its elongation, corresponding to the diameter of the microscopic field of vision. The relation in thickness of the two plates $\left(d_{\mathrm{M}}\right.$ and $\left.d_{\mathrm{N}}\right)$ is as shown in the figure: $d_{\mathrm{M}}<d_{\mathrm{N}}$ toward the thinner edge of the wedge, $d_{\mathrm{M}}>d_{\mathrm{N}}$ toward its thicker edge, and $d_{\mathrm{M}}=d_{\mathrm{N}}$ at a certain intermediate position, $\mathrm{H}$. The compound wedge exhibits a series of retardation, continuously varying from point to point along its elongation, corresponding to the variation in $d_{\mathrm{M}}-d_{\mathrm{N}}$. The retardation is zero at $\mathrm{H}$. Designate the position on the wedge, apart $r$ from $\mathrm{H}$ on its thinner side as $\mathrm{K}$, and the position $1.5 r$ apart from $\mathrm{H}$ on its thicker side as $\mathrm{L}$. The portions of the wedge outside of $\mathrm{K}$ and $\mathrm{L}$ may be cut off.

The optical quality of the compound wedge thus made is given by the diagram in Fig. 2, drawn on the basis of the known optical data of quartz. This is a consequence of the construction as described. In the diagram, $\Delta$ denotes the phase difference in vibration between two emerging light waves due to the wedge. The diagram shows how, in the wedge, $\Delta$ and $d_{\mathrm{M}}-d_{\mathrm{N}}$ are related to each other for light of different wave-lengths. It is seen from the diagram that, for light of any wave- 


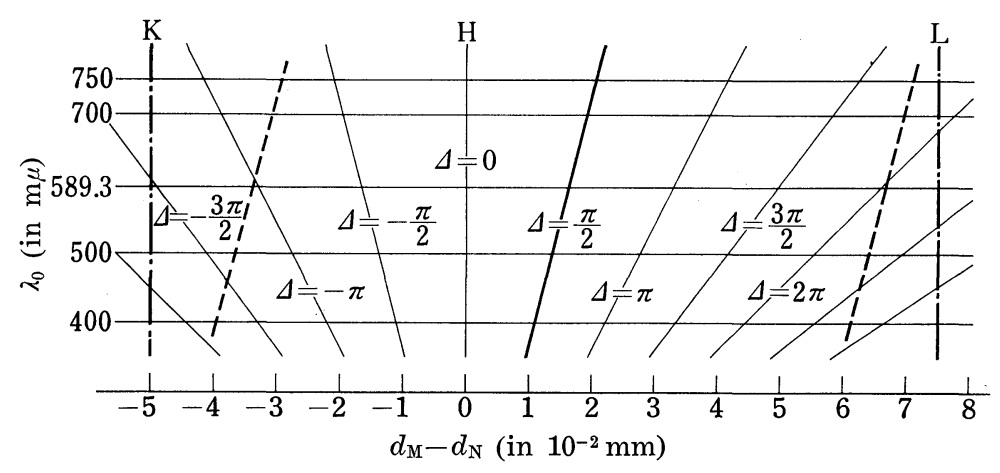

Fig. 2. Optical quality of the compound wedge. The thick full line indicates the location of $\frac{\pi}{2} \Delta$-points on the wedge, and the thick broken lines the edges of the microscopic field of vision when the $\frac{\pi}{2} \Delta$-points are at its center.

length in the range $\lambda_{0}=400-750 \mathrm{~m} \mu$, all of the three locations on the wedge, where $\Delta=-\frac{\pi}{2}, \Delta=\frac{\pi}{2}$, and $\Delta=\frac{3 \pi}{2}$, can be brought simultaneously within a microscopic field of vision. This is a quality, most desirable for general use with the whole range of visible light.

\section{On the Procedure for Retardation Determination}

Of the procedure previously described for determining the retardation of a given crystal section $\mathrm{S}$, the following modification is proposed.

Prior to an examination of the given section S, a preliminary experiment as follows is to be made. It is for locating the point on the wedge where $\Delta=\frac{\pi}{2}$ (designated as $\frac{\pi}{2} \Delta$-point). For this purpose, place the wedge at the proper position in a polarizing microscope in orthogonal orientation ${ }^{1)}$; put any appropriate birefringent crystal plate $\mathrm{C}$, on the microscope-stage in diagonal orientation ${ }^{2)}$; and make an observation through the rotatable analyzer at its appropriate azimuth, with monochromatic light transmitted from below. Then, a row of light and dark bands is seen in the microscopic field of vision. There the bands of different brightness are arranged symmetrically along the elongation of the wedge, and the $\frac{\pi}{2} \Delta$-point constitutes a center of symmetry in the brightness distribution.

The brightness (intensity of light) at any point in the row of bands is given by the relation:

1) With the elongation of the wedge perpendicular to the vibration direction of the polarizer.

2) In the direction $\nwarrow X^{\prime}: \nearrow Z^{\prime}$. 


$$
I^{\prime}=I\left(\sin ^{2} \frac{\delta}{2} \cos ^{2} \zeta+\cos ^{2} \frac{\delta}{2} \sin ^{2} \zeta-2 \sin \frac{\delta}{2} \cos \frac{\delta}{2} \sin \zeta \cos \zeta \sin \Delta\right),
$$

where $I$ denotes the intensity of linearly polarized light incident upon the crystal plate $\mathrm{C}, I^{\prime}$ the intensity of the light reaching to the observer's eye, $\delta$ the phase difference in vibration between two emerging light waves due to the crystal plate $\mathrm{C}, \Delta$ that due to the wedge, and $\zeta$ the azimuthal angle of the analyzer, measured counterclockwise from its initial direction $\leftrightarrow$.

From this relation it is evident that the mode of brightness distribution in the row of bands is dependent on the value of $\delta$ of the crystal plate $\mathrm{C}$, and that use of a plate in which $\delta= \pm \frac{(2 n+1) \pi}{2}$ is best fitted for the present purpose, because a row of bands of maximum contrast in brightness is thereby obtainable. The familiar " $\frac{1}{4} \lambda_{0}$ mica plate" attached to the microscope may be conveniently used here.

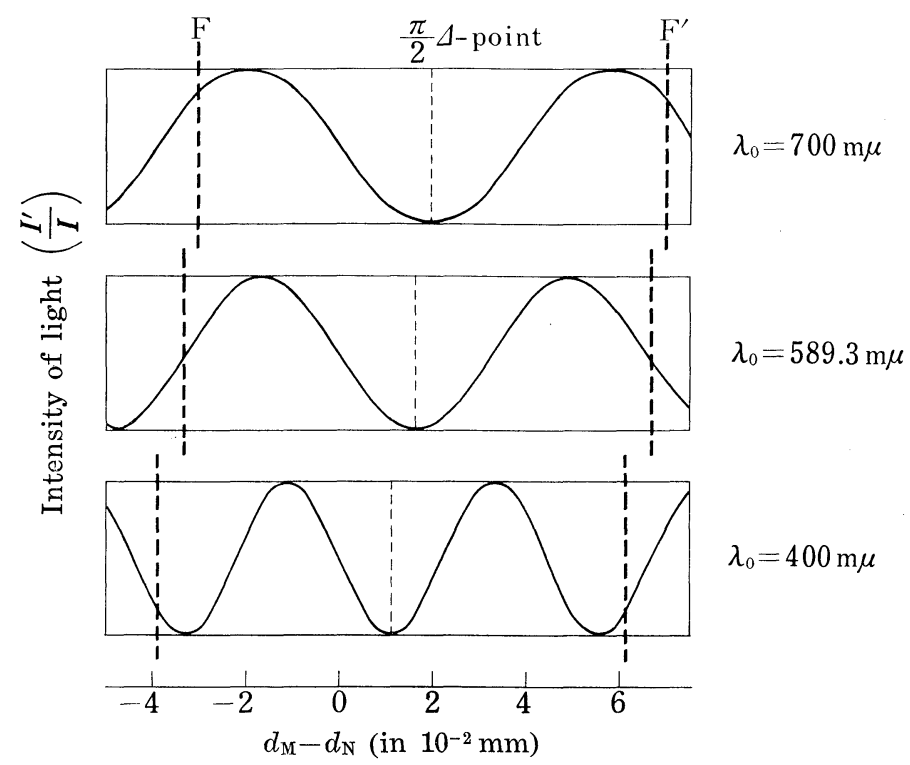

Fig. 3. Brightness distribution in the rows of bands along the elongation of the wedge, obtainable in combination with a plate in which $\delta=\frac{\pi}{2}$, at azimuthal angle $\zeta=45^{\circ}$ of the analyzer. Here the intensity of light is represented by $\frac{I^{\prime}}{I}$. Thick broken lines $\mathrm{F}$ and $\mathrm{F}^{\prime}$ indicate the edges of the microscopic field of vision when the $\frac{\pi}{2} \Delta$-points are at its center.

3) In the plate, the value of $\delta$, depending on the wave-length of the light used, is $\frac{\pi}{2}$ or not far from $\frac{\pi}{2}$. 
The $\frac{\pi}{2} \Delta$-point on the wedge having been thus located, next to do is to bring it to the center of the microscopic field by sliding the wedge and to secure it there. Then, instead of the plate $\mathrm{C}$ put the section $\mathrm{S}$ at the center, oriented in the same way. Thereafter proceed as described in the previous paper.

The procedure, modified as above, is similar to that previously proposed for examination of minute objects. It may be applied with advantage to any objects, irrespective of their size.

The compound wedge, as specified in this paper, is of high efficiency for the above-mentioned preliminary experimentation, because of its optical quality given in Fig. 2. By use of the wedge in combination with a plate in which $\delta=\frac{\pi}{2}$, the brightness distribution as exemplified in Fig. 3 is obtained at azimuthal angle $\zeta=45^{\circ}$ of the analyzer. In the row of bands seen there, both the brightest and darkest bands lie side by side in full contrast within the field of vision. This condition must facilitate an accurate location of the $\frac{\pi}{2} \Delta$-point on the wedge. 